$62^{\text {ème }}$ Congrès de la SFCO, 02002 (2014)

DOI: $10.1051 / \mathrm{sfco} / 20146202002$

C Owned by the authors, published by EDP Sciences, 2014

\title{
Antibioprophylaxie de l'endocardite infectieuse, respect des recommandations de bonnes pratiques, enquête nationale auprès des chirurgiens-dentistes.
}

\author{
Cloitre A', Duval $\mathbf{X}^{2}$, Lesclous $\mathbf{P h}^{1}$ \\ 1 UF de Chirurgie Orale, Service d'Odontologie Restauratrice et Chirurgicale, CHU de Nantes, 1 Place Alexis Ricordeau, BP 84215, 44042, Nantes, FRANCE \\ 2 Service des Maladies Infectieuses et Tropicales, Hôpital Bichat, AP-HP, 46 rue Henri Huchard, 75018, Paris, FRANCE
}

\section{Introduction}

Depuis 2002, les recommandations de bonnes pratiques sur l'Endocardite Infectieuse (EI) ont considérablement évolué, restreignant aujourd'hui l'usage de l'antibioprophylaxie aux seules cardiopathies à haut-risque. À ce jour, aucune preuve scientifique de l'efficacité ou de l'absence d'efficacité de l'antibioprophylaxie de l'El n'existe, et il est donc impératif de surveiller les conséquences de la restriction de son utilisation. Cette surveillance repose d'une part sur l'épidémiologie de l'El dont l'incidence en France est stable, et d'autre part, sur l'évaluation de l'adhésion des professionnels aux recommandations actuelles.

Objectif

Le principal but de cette étude est d'évaluer le respect des recommandations actuelles concernant l'antibioprophylaxie de l'El auprès des chirurgiens-dentistes exerçant en France.

\section{Méthodes}

Une enquête nationale de bonnes pratiques cliniques a été réalisée à partir d'un questionnaire élaboré en collaboration avec l'Association pour l'Etude et la Prévention de l'El. Un sondage électronique, totalisant 136 questions, a été diffusé du 5 septembre au 5 octobre 2012 aux 12000 praticiens adhérents à l'Union Française de Santé BuccoDentaire.

\section{Résultats}

906 praticiens ont répondu au questionnaire et 530 l'ont achevé entièrement. $94 \%$ des chirurgiens-dentistes suivent des patients à risque d'El, et pourtant ils ne sont que $57 \%$ à situer de manière correcte la date de parution des recommandations actuelles. L'étude souligne un manque de connaissance des répondants sur la prise en charge préconisée actuellement des patients à risque d'El, que ce soit pour l'évaluation du risque d'El, sa prévention ou le suivi des sujets prédisposés. Les praticiens ne sont pas conscients de leurs insuffisances : $59 \%$ déclarent avoir une bonne ou une très bonne connaissance des recommandations, mais seuls $13 \%$ savent apprécier correctement le risque d'El des cardiopathies, $3 \%$ connaissent vraiment les gestes autorisés ou contre-indiqués chez un patient à risque d'El, et $22 \%$ seulement ont un schéma de prescription conforme. À ceci se surajoute un manque d'adhésion des praticiens au référentiel en vigueur promu par l'Agence Nationale de Sécurité du Médicament en 2011. Si $57 \%$ des chirurgiens-dentistes sont au courant de sa publication, ils ne sont que $41 \%$ à déclarer avoir modifié leur pratique selon ce référentiel et $16 \%$ à avoir adapté leur fréquence de prescription. Deux facteurs majeurs d'échappement ont été identifiés par cette enquête : le faible niveau de preuve scientifique de ces recommandations et une évolution considérable dans la pathogénèse de l'El. Au final, les trois quart des répondants continuent à prescrire, à tort, une antibioprophylaxie pour les cardiopathies à risque modéré. Néanmoins, en comparant ces résultats avec ceux de deux enquêtes précédentes superposables réalisées en 1991 et 2001, l'application des recommandations s'est globalement améliorée en 20 ans.

\section{Conclusions}

Le respect des recommandations sur la prise en charge des patients à risque d'El est perfectible. L'apparente stabilité des El à streptocoques oraux observée en France depuis la réduction du champ d'application de l'antibioprophylaxie en 2002, pourrait davantage refléter le manque d'adhésion des praticiens que l'efficacité réelle des stratégies de prévention actuelles.

CLOITRE Alexandra

alexandra.cloitre@hotmail.fr

This is an Open Access article distributed under the terms of the Creative Commons Attribution License 4.0, which permits unrestricted use, distribution, and reproduction in any medium, provided the original work is properly cited. 